\title{
Experiencias de trabajo cooperativo en la Universidad Politécnica Salesiana
}

Ana María Narváez*, Jaime Padilla**, María Sol Villagómez***

\section{Antecedentes}

En el marco de las competencias genéricas y los aprendizajes de los docentes en el curso DIDUPS, se propone, desde el Área de Educación, continuar el proceso de reflexión docente sobre la aplicación metodológica del "Aprendizaje cooperativo en el aula". Con el fin de fortalecer las prácticas educativas de los docentes de la UPS a partir del aprendizaje cooperativo, se organizaron dos foros virtuales entre marzo y junio de 2011, cuyos objetivos específicos fueron:

- Comprender y consolidar las concepciones teóricas que se manejan alrededor de este tema.

- Sistematizar las experiencias exitosas de aprendizaje cooperativo en el aula compartidas por docentes de la UPS.

\section{Concepto de 'aprendizaje cooperativo'}

Se trata de un método de aprendizaje que está caracterizado por el trabajo conjunto, fundamentado en el socio-constructivismo. Esta interacción cooperativa se entiende como el trabajo en equipo, es decir, el trabajo colectivo, recíproco y redistributivo (minga) realizado como un 'nosotros', en el que cada uno tiene un rol específico.

El 'aprendizaje cooperativo' difiere del 'aprendizaje competitivo', porque en éste los estudiantes compiten entre sí para lograr los resultados previstos y el rendimiento de un alumno o grupo de estudiantes implica que el rendimiento de los demás sea menor. Difiere también del 'aprendizaje individualista' porque en este el estudiante se centra en la realización de su tarea, independientemente del logro de los demás compañeros.

Entre las características esenciales del aprendizaje cooperativo se incluyen:

\footnotetext{
* Docente de la UPS, Carrera de Pedagogía

** Centro de Investigación Educativa de la UPS

*** Directora del Área de Educación de la UPS
} 
La interdependencia positiva. Es el sentimiento de trabajar en equipo, de estar vinculados entre sí para alcanzar un objetivo común. Si uno de los miembros falla es imposible alcanzar el resultado final. Por una parte, todos necesitan de los demás, pero por otra, cada uno es importante para el logro del resultado.

La interacción estimuladora. Preferentemente 'cara a cara' o simultánea. Los estudiantes tienen que aprender a trabajar juntos, compartiendo conocimientos, recursos y apoyo; deben discutir sobre los distintos puntos de vista $y$ explicar a los demás lo que cada uno va aprendiendo.

La responsabilidad individual. Implica que cada uno sea responsable de contribuir al aprendizaje y al éxito del grupo, pero al mismo tiempo demuestre públicamente su competencia. Esta característica sintoniza y se complementa con la interdependencia positiva.
Las habilidades sociales. Estas habilidades se relacionan con el aprendizaje y las relaciones interpersonales, y los estudiantes deben ser capaces de manejarlas. Estos roles y relaciones implican ejercicio del liderazgo, aceptación o no por el resto de compañeros, la gestión de posibles conflictos, etc. Saber trabajar en equipo es una competencia profesional que hay que aprehenderla.

La autoevaluación del grupo. Es la oportunidad de los estudiantes para evaluar el proceso de aprendizaje. Implica que los alumnos deben aprender a analizarse y analizar a los demás, tanto en el ejercicio individual como grupal.

A partir de los instrumentos que se apliquen, la Universidad Politécnica de Madrid propone las siguientes técnicas para aplicar el método de aprendizaje cooperativo: JIGSAW o rompecabezas (Aronson, 1975), STAD o divisiones de rendimiento por equipos (Slavin, 1978), grupo de investigación (Sharan y Sharan, 1976), COOPCOOP (Kagan, 1985), entre otros.

Gráfico 1

Mentefacto Conceptual de Aprendizaje Cooperativo

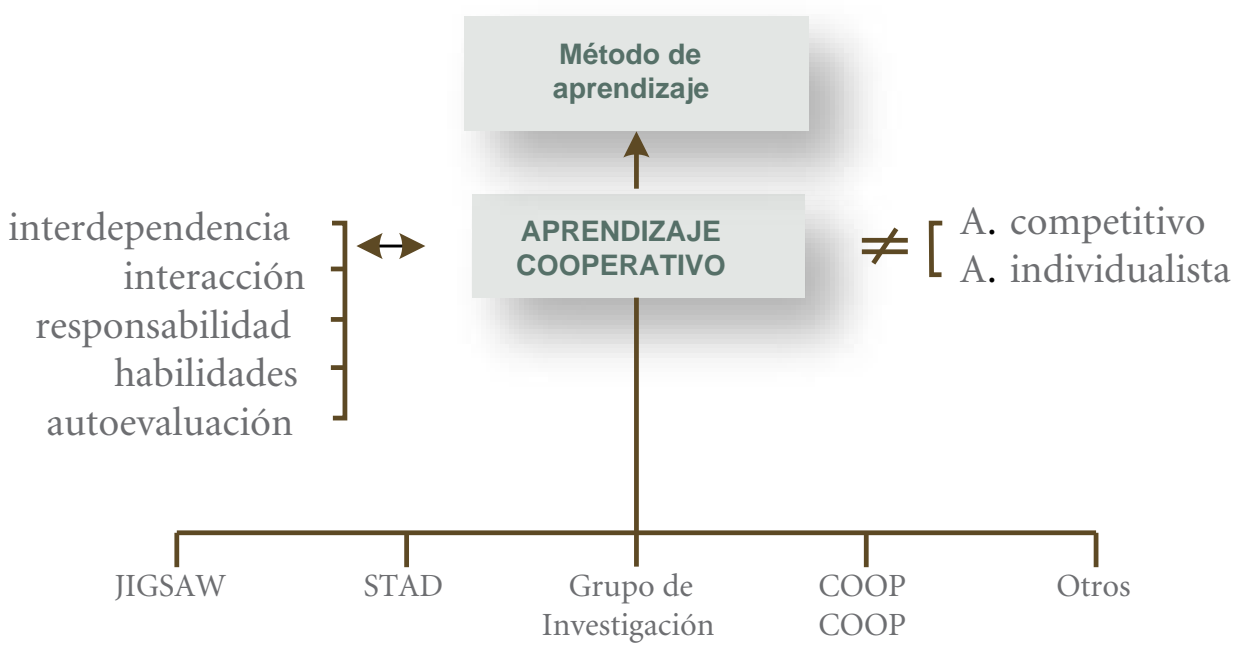




\section{Las experiencias de aprendizaje cooperativo en la UPS}

Las experiencias compartidas por los docentes son expuestas de acuerdo a los siguientes elementos: denominación de la estrategia, asignatura en la que se aplicó la estrategia, objetivos de aprendizaje, proceso de aplicación de la estrategia, recursos y resultados obtenidos. Las participaciones en el foro no siempre siguieron estos lineamientos, por lo que en algunos casos la experiencia no se encuentra descrita en su totalidad.

\section{Docente: Verónica Di Caudo}

Denominación de la estrategia: Aprendiendo Juntos

Asignatura: Evaluación en Nivel Inicial

\section{Objetivo de aprendizaje:}

Transferir conceptualmente la creación de un instrumento de evaluación válido y con posibilidades de ser aplicado.

\section{Proceso de aplicación de la experiencia:}

- La actividad necesita aproximadamente tres horas de clase.

- Los grupos deben ser de 4 estudiantes heterogéneos (con diferentes niveles de rendimiento y competencias), permitiendo el trabajo en parejas.

- Se retoma en un diálogo participativo lo que se trabajó la clase anterior. Sobre la temática de técnicas e instrumentos de evaluación, se recuperan conceptos, se aclara alguna duda y se solicitan ejemplos. Puede utilizarse la estructura 'pienso, intercambio y comparto en grupo', tarjetas, lluvia de ideas o cualquier otra técnica que ayude a revisar lo aprendido.
- Explicación de lo que se hará, formación de grupos, organización del espacio y los materiales.

- Los estudiantes trabajan una hora en grupos, creando y diseñando un instrumento de evaluación.

- Una hora plenaria donde cada grupo muestra su trabajo y los demás interactúan. Se otorga un tiempo máximo por grupo, según su número.

- La plenaria también puede tener la forma de una feria o muestra, colgando los instrumentos de evaluación para que los demás los observen y analicen.

- La hora plenaria se realiza según el número de trabajos a exponerse y el tiempo disponible, teniendo en cuenta que lo planificado no siempre se ajusta a lo ideal.

- Quince minutos para un diálogo evaluativo y metacognitivo. También pueden utilizarse entrevistas a los grupos.

\section{Recursos necesarios:}

- mesas y sillas

- cuatro estudiantes

- documentos referenciales del currículo

- ejemplos de instrumentos curriculares

- texto base

- palógrafos

- marcadores

\section{Resultados obtenidos:}

Consecución de los objetivos de aprendizaje

Docente: Sebastián Araujo

Denominación de la estrategia: Trabajo Comunal Asignatura: Matemáticas y Física 


\section{Objetivo de aprendizaje:}

Aplicar las matemáticas y la física a la resolución de ejercicios.

\section{Proceso de aplicación de la experiencia:}

- Formar grupos de 5 integrantes máximo. Los grupos se forman libremente y se designan autoridades dentro del grupo.

- Se asigna solo un ejercicio a cada grupo. El ejercicio de similar complejidad al que se he resuelto en clase.

- Se revisan y corrigen los ejercicios a medida que cada grupo los concluye.

- El tiempo de presentación del ejercicio termina con el tiempo de la clase.

- No hay deberes para la casa, la tarea consiste en que todos los grupos socialicen los ejercicios resueltos.

\section{Recursos necesarios:}

- Cuaderno, lápiz y calculadora (en el mejor de los casos laptop)

- Movilidad de los pupitres

- Espacio suficiente en el aula

- Libro de ejercicios

\section{Resultados obtenidos:}

- Mayor cohesión dentro del grupo

- Mejores aprendizajes, mayor aprovechamiento

- Se logra un buen nivel de aprendizaje entre todos los estudiantes

\section{Docente: Tania Chicaiza}

Denominación de la estrategia: Proyecto en Grupo Asignatura: Fundamentos de Marketing

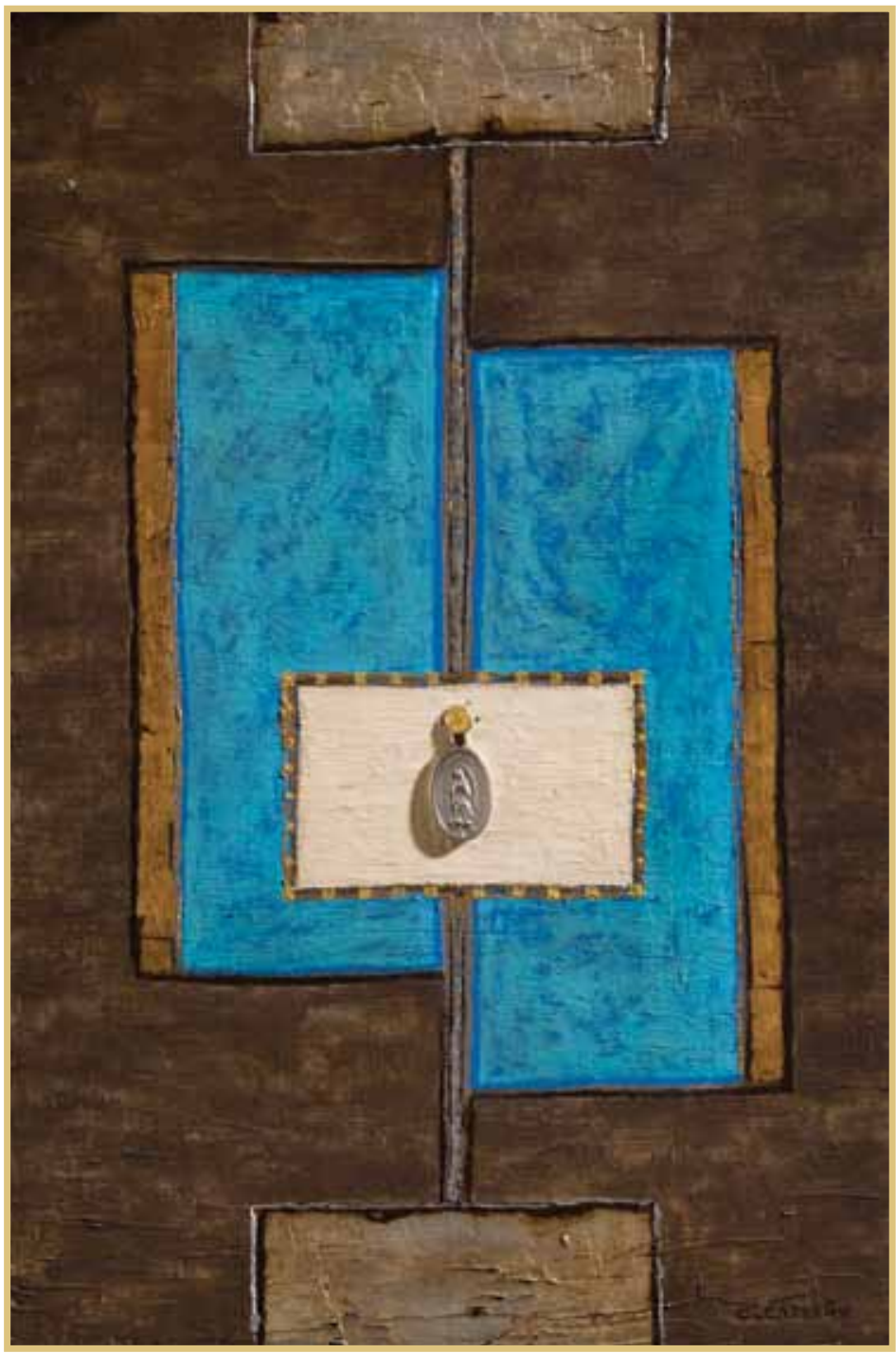

Medalla de Guadalupe

\section{Objetivo de aprendizaje:}

Estructurar un plan de marketing estratégico.

\section{Proceso de aplicación de la experiencia:}

Formación de grupos que no respondan únicamente al criterio de afinidad, sino también a criterios de equidad de género, sectores de donde provienen los alumnos, etc.

Se sortea un grupo que será el que haga seguimiento del trabajo de otro grupo, por tanto, cada nota considera la coevaluación entre los grupos. Se apela a la madurez y calidad de los criterios para mejorar el trabajo. 
Cada vez que se realicen las exposiciones, el grupo evaluador debe presentar un modelo de evaluación (rúbrica) en función del cual emitirá una nota. Esta rúbrica también tiene una calificación.

Todos los grupos deben informar de manera quincenal sobre su trabajo, para evaluar el nivel de compromiso, apoyo y responsabilidad de cada miembro. El ejercicio ayuda a identificar el aporte de cada estudiante y el diálogo generalmente permite identificar a quienes se han deslindado de responsabilidades. Lo interesante es que se genera un espacio de confrontación sin que esto implique retirar a alguien del grupo, más bien se le asignan tareas específicas. Sin embargo, si aun así se retira, el estudiante debe emprender el proyecto solo.

\section{Recursos necesarios:}

- un computador por grupo

- documento base

- guía de trabajo

- afiches

- filmadora

- AVAC de la UPS

\section{Resultados obtenidos:}

A la docente le ha permitido conocer la realidad y capacidad de cada uno de los estudiantes; mientras que ellos han aprendido a colaborar activamente desde muchos ámbitos y a fortalecer la responsabilidad en el grupo.

\section{Docente: Holger Díaz}

Denominación de la estrategia: Mink'a de Aprendizaje

Asignatura: Realidad Nacional e Internacional

\section{Objetivo de aprendizaje:}

Observar en el campo la realidad para analizarla y sistematizarla.

\section{Proceso de aplicación de la experiencia:}

- La mink'a de aprendizaje requiere de un entrenamiento previo, tanto de tipo teórico-conceptual como metodológico. De hecho, existe hoy un gran acumulado de conocimientos teóricos que nos permiten comprender mucho mejor la mink'a de aprendizaje. Sin esta apropiación previa, no es posible poner en acción ningún modelo, metodología u otra aplicación sobre el aprendizaje cooperativo.

- La aplicación en clase de la mink'a de aprendizaje (orden metodológica) requiere de una fuerte planificación (léase como mentalidad proyectual), organización, aplicación y evaluación del objetivo de aprendizaje.

- La planificación está relacionada con el programa de estudios y con los objetivos de aprendizaje del programa analítico. Cada uno de estos objetivos se traduce en una planificación de temáticas de estudio con sus respectivos componentes didácticos.

- La organización tiene que ver con la manera en que se generan equipos de trabajo para lograr un mismo objetivo de aprendizaje. Generalmente, para el aula de clase de esta materia, se organizan equipos de investigación de campo. Los equipos pueden estar conformados entre 3 y 5 personas. La conformación de los equipos de investigación se realiza al azar o por afinidad. El principio de afinidad hace que los alumnos compartan unos mismos intereses y deseos.

- Una vez que los alumnos han realizado la investigación (de campo o bibliográfica) deben socializar los resultados de la misma a través de exposiciones en clase. 
Cada equipo tiene entre 10 y 15 minutos para exponer su investigación. Además, el profesor y los alumnos pueden plantear preguntas relacionadas con el tema para que sean aclaradas por el grupo expositor.

- Otra manera de hacer mink'a de aprendizaje en clase es través de mesas redondas. Se distribuye a los alumnos por grupos de afinidad para reflexionar o discutir algún tema relacionado con el objetivo de aprendizaje. Cada mesa se configura entre 3 a 5 miembros, quienes debe elegir un coordinador y un asistente o secretario, el cual se encarga de anotar la información generada por el grupo para luego ser expuesta a través de un foro público en la clase. El trabajo de las mesas redondas se expone a través de cada uno de los asistentes o secretarios. El foro público consiste en una discusión respecto del tema reflexionado en cada mesa redonda.

- Otra manera que coadyuva a la mink'a de aprendizaje es la técnica "Jurado No.13". Se trata de imitar un jurado real compuesto por 12 actores más la participación total del curso. Los actores del jurado son: un juez y su secretario, dos fiscales como mínimo y cuatro testigos por cada bando de discusión. Se denomina "Jurado No.13" porque el veredicto final lo da el curso, en calidad de último juez.

- Otra técnica interesante es el panel, que busca reflexionar, discutir y encontrar soluciones o alternativas viables a algún problema relacionado con la temática que se aborda en clase. El mecanismo es el mismo que se usa en los foros académicos públicos de la Universidad.

\section{Recursos necesarios:}

Dependerán de los objetivos de aprendizaje, por ejemplo: cámara fotográfica, cámara de video, instrumentos de registro de las observaciones.

\section{Resultados obtenidos:}

- capacidad para la investigación de campo y bibliográfica

- análisis de la realidad

- capacidad para trabajar en equipo

- capacidades de comunicación escrita y oral

\section{Docente: Eduardo Morán}

Denominación de la experiencia: La Reja

Asignaturas: Psicoterapias Humanistas y Psicoterapia Gestáltica

\section{Objetivo de aprendizaje:}

Conocer y manejar comprensivamente contenidos diversos relacionados con un mismo tema utilizando la metodología cooperativa.

\section{Proceso de aplicación de la experiencia:}

- Dividir al grupo en subgrupos. Cada subgrupo tiene un número de estudiantes múltiplo del número total, por ejemplo, si tengo 35 alumnos los divido en siete grupos de cinco.

- A cada subgrupo se le entrega una parte del tema o subtema.

- Se ofrece una explicación introductoria del tema y se explica el desarrollo de la estrategia de 'la reja'.

- Cada subgrupo estudia un subtema asumiendo que cada integrante está en capacidad de transmitir ese contenido, de la forma más fidedigna posible, al resto de participantes del gran grupo.

- Se asigna a cada estudiante una letra, habrán tantas letras como el número de grupos que se hayan conformado.

- Se organizan en un segundo subgrupo, el cual está compuesto por un representante 


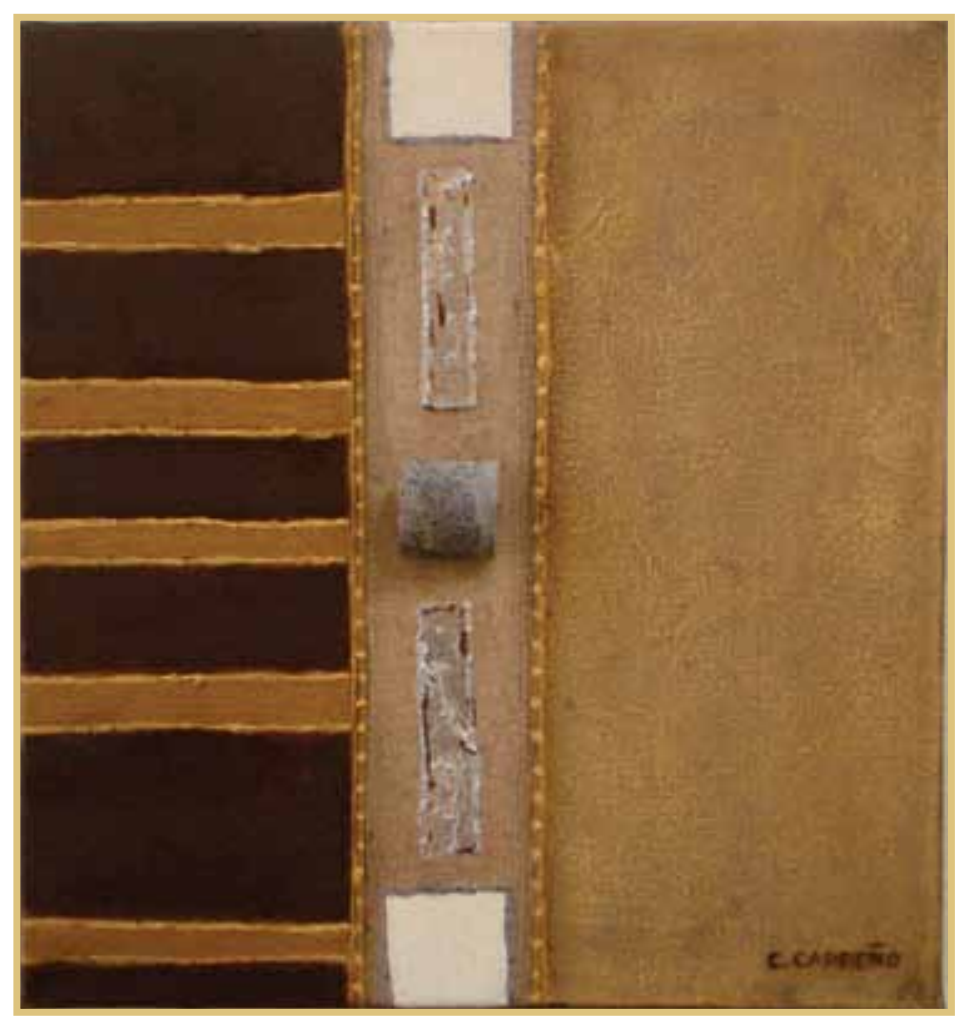

ST de los grupos anteriores (organizados por números).

- Cada estudiante comunica a los compañeros lo aprendido en el subtema asignado (al grupo por números). Así se complementa la información global del tema.

- El docente va de grupo en grupo escuchando cómo estudian y cómo comunican los contenidos. Estas observaciones sirven para retroalimentar al grupo, resaltar los puntos ya asimilados y reforzar aquellos que han quedado poco comprendidos o expuestos.

- La actividad se complementa con un foro donde participa un representante del segundo subgrupo (organizado por letras) y el gran grupo hace las preguntas que considera pertinentes.

\section{Recursos necesarios:}

- motivación previa

- cinco capítulos de un mismo tema (uno para cada grupo)

- salón en donde se puedan reunir cinco o más grupos para dialogar en círculos

- dos horas continuas

\section{Resultados obtenidos:}

- Cobertura de los diversos aspectos, componentes, factores, enfoques y teorías de un mismo tema para ser conocido, estudiado, asimilado y manejado con fines aplicativos.

- Se logra un momento didáctico, previo a la discusión grupal, en la cual caben otras aplicaciones metodológicas para lograr que la teoría sea orientada a la aplicación en psicoterapia.

- Celeridad en el estudio de un mismo tema por todo el curso. 
- Disminución de la resistencia a participar con otros compañeros.

- Oportunidad para utilizar la habilidad para atender, concentrarse y percibir los contenidos de un documento con la ayuda de las percepciones de los demás.

- Preparación anímica y mental para tratar sobre la psicoterapia gestáltica, cuyo principio más importante dice que "el todo es más que la suma de las partes", y que un contenido comprendido es "una forma" sobre "un fondo" que es el ambiente o los demás conocimientos o formas de pensar que ya poseemos.

- Oportunidad de entender que el grupo necesitaba una experiencia que lo conduzca, más allá del entendimiento intelectual, a una comprensión existencial del manejo de lo que es la psicoterapia gestáltica humanista.

- Para evaluar y reforzar los conocimientos desarrollados, se concluyó con una síntesis complementaria (puede hacerse una mesa redonda, un ensayo individual, unmapa conceptual, etc.), que dio paso a la utilización del método Dentro-Fuera y a un foro apoyado en los AVAC.

- Si bien la evaluación del uso de 'la reja' incluye la funcionalidad de este método, el modo de uso, el grado de participación y la responsabilidad de los diversos participantes, el foco más importante de la evaluación se centra en la comprensión, análisis deductivo, reflexión, relacionalidad, generalizaciones $\mathrm{y}$ aplicaciones encontradas en el desarrollo tanto de esta técnica como del método Dentro-Fuera y el foro en los AVAC.

\section{Docente: Eduardo Morán}

Denominación de la experiencia: Técnica DentroFuera

Asignaturas: Psicoterapias Humanistas y Psicoterapia Gestáltica

\section{Objetivo de aprendizaje:}

Experimentar y comprender en grupo contenidos que ya han sido revisados en el estudio teórico, utilizando metodología de aprendizaje cooperativo.

\section{Proceso de aplicación de la experiencia:}

- Días antes los alumnos estudian dos documentos que aclaran diversos conceptos, principios, técnicas y procedimientos de la psicoterapia gestáltica, enviados a través de los AVAC.

- El día convenido se parte de una síntesis de elementos comprendidos y de cuestionamientos surgidos en el estudio teórico.

- Se explica el procedimiento a seguir.

- Se divide en dos subgrupos, cada uno conformado por el $50 \%$ de la lista.

- Cada grupo elige un coordinador que dé la palabra, invite a participar o limite a quienes lo hacen muchas veces.

- Se sientan en dos círculos concéntricos, uno dentro y otro fuera.

- Quienes se hallan 'dentro' dialogan sobre los contenidos estudiados, usando la técnica de 'la reja', mientras quienes se hallan 'fuera' observan y escuchan el desarrollo del diálogo en silencio.

- Luego de algunos minutos, los grupos cambian de posición. Quienes estaban 'fuera' entran al diálogo y quienes estaban 'dentro' pasan a hacer silencio para escuchar a sus compañeros.

- El docente motiva la participación, la profundización de lo manifestado en las discusiones de ambos grupos y la confrontación utilizando diversas técnicas de psicoterapia gestáltica y estableciendo relación entre un estudiante (forma) y el grupo (fondo), para llevarles a "vivir el aquí y el ahora" y "darse cuenta" de la dificultad de comprender, aceptar o asumir una técnica. 
Solo así podrán entender lo que es 'fondo' y lo que es 'forma'.

- Este aprendizaje se evalúa a partir de una pregunta motivadora en el foro realizado por los estudiantes a través del AVAC abierto por cinco días.

\section{Recursos necesarios:}

- motivación previa

- contenidos que deben ser estudiados previamente (uno o varios capítulos)

- salón donde puedan conformar dos círculos concéntricos

- dos horas continuas

\section{Resultados obtenidos:}

- Plena comprensión desde lo experiencial, más allá de los conocimientos teóricos, para aplicar en sí mismos estos procesos de meta-aprendizaje, para la comprensión de sus limitaciones de pensamiento, para identificar sus mecanismos de defensa, para verificar sus modos de percibir y relacionarse, y para comprender las formas de aplicación a sus casos.

- Corresponsabilidad en el aprendizaje y aprendizaje cooperativo.

- Utilización de habilidades de organización, respeto al pensamiento diverso, respuestas apropiadas a las expresiones de los otros estudiantes, capacidad de escucha.

- Los estudiantes pudieron comprender a través de la experiencia los conceptos del 'aquí' y el 'ahora', el 'fondo' y la 'forma', y los productos nuevos que ocurren en la interrelación, cuyo resultado es más que la suma de las partes. También pudieron asumir reglas de la psicoterapia gestáltica como: cambiar una pregunta a una afirmación, establecer relación directa entre el 'yo' (que se comunica) y el 'tú' (al que se comunica), hablar en presente ('siento que...') y de manera personalizada ('yo creo', 'cuando me ocurre esto yo...'), relacionarse en lugar de hacer disquisiciones filosóficas, comunicar en todo momento, hablar directamente en lugar de murmurar. Asimismo, lograron la aplicación de algunas técnicas como: la silla vacía, representación de roles y la contratransferencia como recurso, entre otras.

- Motivación para aplicar las reglas, técnicas, procedimientos y formas de comunicación y de relación a sus prácticas comunitarias.

- Celeridad en la comprensión de la psicoterapia gestáltica.

- Disminución de la resistencia a participar con otros compañeros y del tiempo utilizado para el estudio.

\section{Bibliografía}

- GÓMEZ, Juan, Aprendizaje cooperativo: metodología didáctica para la escuela inclusiva, La Salle, 2007, versión digital de:

- http://www.lasalle.es/arlep/es/cpropio/documentacion/documentos/Documentos/ Aprendizaje\%20Cooperativo.pdf

- UNIVERSIDAd POLITÉCNICA DE MADRID, Aprendizaje cooperativo, 2008, versión digital de:

- http://innovacioneducativa.upm.es/guias/ Aprendizaje_coop.pdf

- UNIVERSIDAD POLITÉCNICA SALESIANA, Foro General: Aprendizaje Cooperativo $y$ Experiencias Exitosas, aula virtual: trabajo cooperativo en el marco de la diversidad, 2011. 